\title{
Effects of Toe Grasp Training for the Aged on Spontaneous Postural Sway
}

\author{
Ryuji Kobayashi ${ }^{1)}$, Masataka Hosoda ${ }^{1)}$, Akira Minematsu'1), Hisato Sasaki ${ }^{1)}$, \\ Hiroshi Maejima ${ }^{2)}$, Sachiko Tanaka ${ }^{1)}$, Naohiko Kanemura ${ }^{1)}$, Akihisa Matsuo ${ }^{1)}$, \\ Kunji Shirahama ${ }^{1)}$, Takehito Ueda ${ }^{1)}$, Chie Kamoda ${ }^{1)}$, Osamu Yoshimura ${ }^{2)}$ \\ ${ }^{1}$ Student, Health Sciences Major, Graduate School of Medical Sciences, Hiroshima University \\ School of Medicine, 1-2-3 Kasumi, Minami-ku, Hiroshima 734-8551, Japan. \\ $T E L+8182-257-5433$ \\ ${ }^{2}$ Institute of Health Sciences, Hiroshima University School of Medicine
}

\begin{abstract}
Falls are a common problem among elderly persons, but the training for the fall prevention is not well established. Therefore, the effectiveness of toe grasp training was examined in this study. Spontaneous postural sway was tested on elderly persons $(\mathrm{N}=19)$ with eyes open and eyes closed. Using a force plate, sway responses were quantified in terms of the total track length, the environmental areas, the maximum amplitude distance of $\mathrm{X}$-axis, and the maximum amplitude distance of $\mathrm{Y}$-axis. The data was compared between the toe grasp training group and the control group. Total track length (eyes open and eyes closed), environmental areas (eyes open) and maximum amplitude distance of X-axis (eyes open and eyes closed) were improved significantly in the toe grasp training group. The activation of foot mechanoreceptors and improvements of the eye-leg coordination were thought to be factors in this improvement and toe grasp training is expected to be effective for fall prevention of the aged.
\end{abstract}

Key words: Toe grasp training, Spontaneous postural sway, Aged.

(This article was submitted Mar. 31, 1999, and was accepted May. 20, 1999)

\section{INTRODUCTION}

About one third of the aged residing at home are said to have experienced one or more falls, and ten percent of them are said to have suffered bone fractures ${ }^{1,2)}$. In particular, eighty percent of hip fractures result from falls ${ }^{3)}$. A year after the date of fracture, the death rate for those who have suffered a fracture increases by three times compared with those who have not experienced fracture4). Even if the fall does not actually result in a fracture, it triggers the fear of falling. Subsequently, such trauma may restrict elderly persons from engaging in their daily activities ${ }^{5}$.

Dangerous factors leading to a fall include physiological, cognitive, and environmental factors. Physical adjustment to the environment factor is now taken as a major preventive approach against a fall. However, with the aging of society and an increase in the life span, other alternative approaches should be considered in dealing with this problem.

According to Ihara6), toe grasp training increased the postural adjustment function in an experiment involving university students. The training is simple and safe, so it is safely applicable even to the aged. Thus, in this experiment, we examined if the training can actually prevent a fall by evaluating the amount of spontaneous postural sway, while standing, occurring at the center-of-gravity (center of foot pressure).

\section{METHOD}

Thirty (15 men and women) nursing home resi- 
dents were selected as subjects for this experiment. The criteria of these test subjects was that they were neither affected with paralysis nor dementia and could stand still in a stationary upright position for one minute or more. Before the experiment was conducted, the purpose and content of the research was explained to each subject, and their agreement to participate in the study was obtained. The subjects were randomly divided into the training group and the control group. By confirming that there was no difference in the spontaneous postural sway measurement result and the average age of the test subjects, the training began.

\section{Toe grasp training}

For the training group, the following training using the toes was executed for 10 minutes, 3 times a week for 8 weeks: i) gathering a towel attached to a weight (Fig. 1-A); ii) passing the bean bags from one place to another (Fig. 1-B). While this particular activity was conducted on the training group, the control group participated in group physical exercise instead. For reasons of defective physical conditions and discharge, 9 people from the training group (average age, $77.8 \pm 2.8$ years old; 3 men and 6 women) and 10 people from the control group (average age, $76.78 \pm 5.2$ years old; 6 men and 4 women) were able to complete the experiment.

\section{Spontaneous postural sway measurement}

The spontaneous postural sway was measured by posturography with a Gravicorder GS-10 (Anima Co.) before and after the training. Each of the test subjects was asked to stand with both feet, bared, set apart by $20 \mathrm{~cm}$, measured from the inside of each foot. Using a sampling frequency of $20 \mathrm{~Hz}$ carried out for $20 \mathrm{sec}$, the measurement was conducted for the following two cases: i) when each subject stared at an object $1.5 \mathrm{~m}$ ahead with the eyes open, and ii) after the oscillation subsided under a condition of the eyes closed while the subject wore an eye mask. This measurement was performed three times respectively with breaks in between, and the average values thus obtained were adopted as the measured data.

The parameters of the experiment included: total track length of open eyes (LNG-O) and total track length of closed eyes (LNG-C); the environmental areas of open eyes (ENV-O) and the environmental areas of closed eyes (ENV-C); maximum ampli-
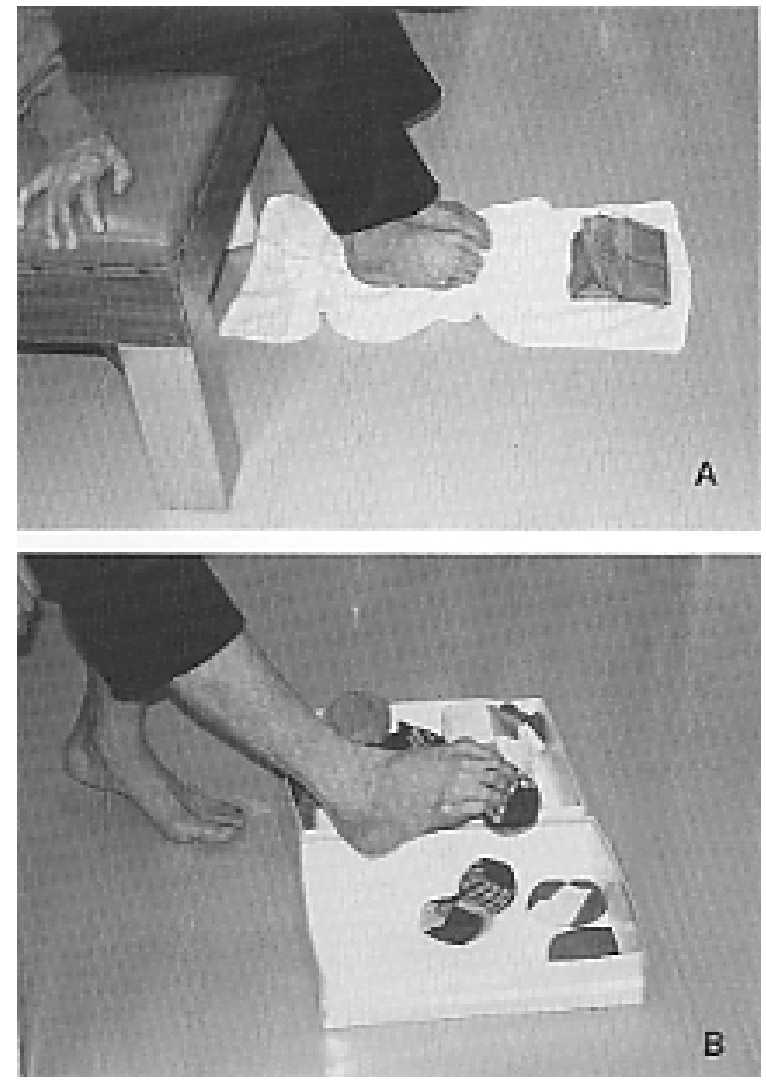

Fig. 1. Toe grasp training. A: Gathering a towel attached to a weight, B: Passing bean bags.

tude distance of X-axis of open eyes (XD-O) and maximum amplitude distance of $\mathrm{X}$-axis of closed eyes (XD-C); maximum amplitude distance of $\mathrm{Y}$ axis of open eyes (YD-O) and maximum amplitude distance of Y-axis of closed eyes (YD-C). In reference to the data taken from the 19 subjects completing the experiment, an average of each test item, in respect to each target group, was calculated by using the corresponding t-test. Results were considered statistically significant when the $P$ value was 0.05 or less. Table calculation software (Microsoft Excel 98 for Macintosh) was used for statistical processing.

\section{RESULTS}

\section{Data comparison of control group (Table 1)}

Significant differences were seen in the following test items: total track length of closed eyes (pre-training $27.65 \pm 6.82 \mathrm{~cm}$; post-training 33.49 $\pm 8.15 \mathrm{~cm}$ ) and environmental areas of open eyes 
Table 1. Data comparison of control group

\begin{tabular}{lrcc}
\hline & pre-training & post-training & p-value \\
\hline LNG-O $(\mathrm{cm})$ & $21.31 \pm 4.32$ & $24.54 \pm 5.79$ & N.S. \\
LNG-C $(\mathrm{cm})$ & $27.65 \pm 6.82$ & $33.49 \pm 8.15$ & $\mathrm{P}<0.01$ \\
ENV-O $\left(\mathrm{cm}^{2}\right)$ & $0.33 \pm 0.23$ & $0.54 \pm 0.30$ & $\mathrm{P}<0.05$ \\
ENV-C $\left(\mathrm{cm}^{2}\right)$ & $0.39 \pm 0.21$ & $0.51 \pm 0.29$ & N.S. \\
XD-O $(\mathrm{cm})$ & $0.80 \pm 0.34$ & $0.95 \pm 0.24$ & N.S. \\
XD-C $(\mathrm{cm})$ & $1.72 \pm 0.41$ & $2.03 \pm 0.30$ & N.S. \\
YD-O $(\mathrm{cm})$ & $0.83 \pm 0.32$ & $0.98 \pm 0.52$ & N.S. \\
YD-C $(\mathrm{cm})$ & $2.32 \pm 0.62$ & $2.44 \pm 0.58$ & N.S. \\
\hline
\end{tabular}

Mean $\pm \mathrm{SD}, \mathrm{N}=10$. LNG: total track length, ENV: environmental area, XD: maximum amplitude distance of $\mathrm{X}$-axis, YD: maximum amplitude distance of $\mathrm{Y}$-axis, O: eyes open, C: eyes closed.

Table 2. Data comparison of training group

\begin{tabular}{lccc}
\hline & pre-training & post-training & p-value \\
\hline LNG-O $(\mathrm{cm})$ & $26.18 \pm 7.26$ & $21.94 \pm 5.14$ & $\mathrm{P}<0.05$ \\
LNG-C $(\mathrm{cm})$ & $35.13 \pm 13.89$ & $25.83 \pm 7.18$ & $\mathrm{P}<0.05$ \\
ENV-O $\left(\mathrm{cm}^{2}\right)$ & $0.99 \pm 0.75$ & $0.41 \pm 0.24$ & $\mathrm{P}<0.05$ \\
ENV-C $\left(\mathrm{cm}^{2}\right)$ & $1.02 \pm 0.97$ & $0.57 \pm 0.71$ & N.S. \\
XD-O $(\mathrm{cm})$ & $1.27 \pm 0.46$ & $0.87 \pm 0.21$ & $\mathrm{P}<0.01$ \\
XD-C $(\mathrm{cm})$ & $2.51 \pm 0.76$ & $1.90 \pm 0.67$ & $\mathrm{P}<0.01$ \\
YD-O $(\mathrm{cm})$ & $1.44 \pm 0.86$ & $1.39 \pm 1.09$ & N.S. \\
YD-C $(\mathrm{cm})$ & $2.59 \pm 1.14$ & $2.30 \pm 0.85$ & N.S. \\
\hline
\end{tabular}

Mean $\pm \mathrm{SD}, \mathrm{N}=9$. LNG: total track length, ENV: environmental area, $\mathrm{XD}$ : maximum amplitude distance of $\mathrm{X}$-axis, YD: maximum amplitude distance of $\mathrm{Y}$-axis, $\mathrm{O}$ : eyes open, $\mathrm{C}$ : eyes closed.

(pre-training $0.33 \pm 0.23 \mathrm{~cm}^{2}$; post-training $0.54 \pm$ $0.30 \mathrm{~cm}^{2}$ ). Each of the results showed an increase in the numerical value.

\section{Data comparison of training group (Table 2)}

Significant differences were seen in the following test items: total track length of open eyes (pre-training $26.18 \pm 7.26 \mathrm{~cm}$; post-training 21.94 $\pm 5.14 \mathrm{~cm}$ ); total track length of closed eyes (pretraining $35.13 \pm 13.89 \mathrm{~cm}$; post-training $25.83 \pm$ $7.18 \mathrm{~cm}$ ); environmental areas of open eyes (pretraining $0.99 \pm 0.75 \mathrm{~cm}^{2}$; post-training $0.41 \pm 0.24$ $\mathrm{cm}^{2}$ ); maximum amplitude distance of $\mathrm{X}$-axis of open eyes (pre-training $1.27 \pm 0.46 \mathrm{~cm}$; post-training $0.87 \pm 0.21 \mathrm{~cm}$ ); maximum amplitude distance of X-axis of closed eyes (pre-training $2.51 \pm 0.76$ $\mathrm{cm}$; post-training $1.90 \pm 0.67 \mathrm{~cm}$ ). Overall, a decrease in the numerical value was seen in each test item.

No significant difference was seen between the change rate of open and closed eyes.

\section{DISCUSSION}

There is a significant correlation between spontaneous postural sway and a fall. It is documented that postural imbalance is a major factor for fall$\mathrm{ers}^{7,}{ }^{8)}$. From the results, it can be seen that the values of total track length and the environmental areas have decreased in the training group. In addition, the results show that the training group had an improvement in the spontaneous postural sway.

Maki et al. ${ }^{9)}$ assumed that the maximum amplitude distance of $\mathrm{X}$-axis is a factor that forecasts a fall in the future. The results this time show better influence on the maximum amplitude for the Xaxis than the Y-axis, thereby suggesting that toe grasp training for the aged could be used as a preventive strategy against a fall.

Ihara $\left.{ }^{6}\right)$ observed that toe grasp training triggers the mechanoreceptor located at the bottom of the foot and the muscular reaction of the lower limbs, 
leading to an improvement in postural adjustment. Physical, visual, and vestibular senses are the necessary requirements for maintaining postural balance, but the aged become more dependent on sight as they become much older ${ }^{10)}$. Such approaches to the physical senses are effective for the aged, and a factor in the improvement of eye-leg coordination might result from toe group training, since in the current results, there were no apparent differences the closed eye condition and the open eye condition.

Furthermore, to carry out the basic training done in this study on a clinical basis for a long period of time is difficult, thus the following three-step training has been implemented:

Pre-training Exercise: There is a decreasing flexibility of the ankle and toe joints seen in the aged. They are instructed to turn their ankles, but extra attention is necessary for those whose lower limbs turn at the same time. For these people, transitive movement such as self-transitive joint exercise and physical therapy should be conducted.

Basic Training: Have individual subjects engage in activities such as pulling the towel and passing the bean bags individually.

Practical Training: Have individual subjects engage in activities such as tug-of-war (using a towel), passing bean bags, and snake lunge (throwing of the bean bags) in a form of game or group. It is difficult to hold this training for a long time unless it is implemented as a part of therapeutic recreational activity or occupational therapy.

In conclusion, further clinical trials should be conducted to show whether toe grasp training can be used as a preventive measure against a fall. In particular, Horak et al. ${ }^{11)}$ attached importance to the dynamic postural sway as a predictive fall factor rather than the spontaneous postural sway.
Hosoda et al. ${ }^{12,13)}$ also observed that footwear is a factor influencing postural balance and should not be overlooked. It will be necessary to consider these criteria in future studies.

\section{REFERENCES}

1) Yasumura $S$, et al: Falls and fractures in the elderly. Journal of Clinical Rehabilitation 2: 707-710, 1993 (in Japanese).

2) Yasumura $S$, et al: Risk factors for falls among elderly people. Rigaku Ryouhou 14: 199-205, 1997 (in Japanese).

3) Hashimoto T, et al: Epidemiology and prevention of hip fracture. Rigaku Ryouhou 14: 206-211, 1997 (in Japanese).

4) Matsubayashi Y: Mortality after hip fracture. Sogo Reha 26: 641-643, 1998 (in Japanese).

5) Howland J, et al: Fear of falling among the community-dwelling elderly, Journal of Aging and Health 5: 229-243, 1993.

6) Ihara H: Dynamic joint control training second edition. Kyodo-isyo press, Tokyo, 1996 (in Japanese).

7) Overstall PW, et al: Falls in the elderly related to postural imbalance. Br Med J 1: 261-264, 1977.

8) Fernie GR, et al: The relationship of postural sway in standing to the incidence of falls in geriatric subjects. Age Aging 11: 11-16, 1988.

9) Maki BE, et al: A prosrective study of postural balance and risk of falling in an ambulatory and imdependent elderly population. Journal of gerontology Medical Sciences 49: M72-M84, 1994.

10) Whipple R, et al: Altered sensory function and balance in older persons. J Gerontol 48: 71-76, 1993.

11) Horak FB, et al: Component of postural dyscontrol in the elderly: A review. Neurobiol Aging 10: 727-738, 1989.

12) Hosoda M, et al: The effect of various footwear types and materials, and of fixing of the ankles by footwear, on upright postural control. J Phys Ther Sci 9: 47-51, 1997.

13) Hosoda M, et al: The effects of footwear on standing postural control. J Phys Ther Sci 10: 47-51, 1998. 